\title{
Genome-wide Analysis of Acute Inflammatory and Anti-Inflammatory Responses in RAW264 Cells Suggests cis-Elements Associated with Translational Regulation
}

\section{Hiroaki Sako ${ }^{1,2}$ and Katsuhiko Suzuki ${ }^{2,3 *}$}

${ }^{1}$ Japan Society for the Promotion of Sciences, Tokyo, Japan

${ }^{2}$ Faculty of Sport Sciences, Waseda University, Saitama, Japan

${ }^{3}$ Institute for Nanoscience and Nanotechnology, Waseda University, Tokyo, Japan

\begin{abstract}
Translational regulation plays pivotal roles in mediating inflammatory responses. Glucocorticoids, represented in this study by dexamethasone (DEX), are widely recognized anti-inflammatory agents that exert significant inhibitory effects on the translation of diverse gene groups, including inflammatory genes. However, their regulation is highly complex and diverse, involving transcriptional and translational regulation. Although transcriptional regulation has been investigated by genome-wide transcriptome analyses, translational regulation has been studied by only a few specific gene targets (e.g., Tumor necrosis factor) and the global impact of glucocorticoids on translation levels has scarcely been studied, mainly due to its technical difficulty. Here, using ribosome profiling coupled with high-throughput mRNA sequencing (mRNA-Seq) in which footprints of translating ribosomes can be captured, we conducted a genomewide transcriptional and translational analysis of the acute inflammatory or anti-inflammatory responses of RAW264 cells stimulated by lipopolysaccharide (LPS) or LPS coupled with DEX (LPS + DEX). We showed that the majority of the differential regulation between LPS alone and LPS + DEX were predominated by translation levels rather than transcription levels. Further analysis on the up- and down-regulated gene clusters revealed putative cis regulatory elements exclusively enriched in the 3'-UTR of either up- or down-regulated genes induced by LPS + DEX. The results imply an alternative to the currently recognized mechanisms of glucocorticoid-induced translational regulation in the acute inflammatory response of RAW264 cells.
\end{abstract}

Keywords: Translational regulation; Glucocorticoid; Dexamethasone; Lipopolysaccharide; RAW264; Genome-wide analysis

\section{Abbreviations:}

LPS: Lipopolysaccharide; DEX: Dexamethasone; mRNA-Seq: mRNA Sequencing; GR: Glucocorticoid Receptor; NF- $\kappa B$ : Nuclear Factor kappa B; Il: Interleukin; IкBa: Nuclear factor of kappa light polypeptide gene enhancer in B-cells inhibitor alpha; Dups1: DualSpecificity Mitogen-Activated Protein Kinase (MAPK) Phosphatase 1; Cxcl1: Chemokine (C-X-C motif) Ligand 1; RBP: RNA Binding Protein; miRNA: microRNA; HuR: Hu antigen R, TTP: Tristetraprolin; ARE: Adenylate-Uridylate-Rich Elements; UTR: Untranslated Regions; Tnf- $\alpha$ : Tumor necrosis factor alpha; Ribo-Seq: Ribosome Profiling; RPF: Ribosome-Protected Fragments; CHX: Cycloheximide; RPM: Reads per Million; nt: Nucleotides; TMM: Trimmed Mean of M; KS: Kolmogorov-Smirnov; GO: Gene Ontology

\section{Introduction}

Glucocorticoids such as dexamethasone (DEX) are potent inflammatory inhibitors and are used in the frontline treatments of various inflammatory diseases. The regulatory mechanisms of DEX or glucocorticoids have been intensively investigated and have been revealed to function in a diverse range, including transcriptional and post-transcriptional regulation.

At transcriptional levels, glucocorticoids bind to the glucocorticoid receptor (GR), residing in the cytoplasm as an inactive form. This interaction triggers the GR translocalization into the nucleus and modulates the target gene expression [1] via physically preventing the activation of nuclear factor kappa B (NF- $\kappa \mathrm{B})$ [2] or activating anti-inflammatory gene expression, including interleukin (Il)-10, Il-1 receptor antagonist, and nuclear factor of kappa light polypeptide gene enhancer in B-cells inhibitor alpha (I $\kappa \mathrm{B} \alpha)$ [3]. Dual-specificity mitogen-activated protein kinase (MAPK) phosphatase 1 (Dusp1) is also known as an activated GR-induced transcriptional suppressor. Dups 1 expression is boosted immediately after the induction of DEX and suppresses MAPK signaling and downstream inflammatory transcriptional events, including those of chemokine ( $\mathrm{C}-\mathrm{X}-\mathrm{C}$ motif) ligand 1 (Cxcl1), Il-6, and Il-8 [4,5].

Post-transcriptional regulation can also exert vital roles in the effect of glucocorticoid-mediated immunosuppression. There are various post-transcriptional controls, involving RNA binding protein (RBP) and microRNA (miRNA), resulting in mRNA destabilization and translational regulation [6]. RBP affects both directions of mRNA stability, represented by a generally positive regulator of $\mathrm{Hu}$ antigen $\mathrm{R}(\mathrm{HuR})$ and tristetraprolin (TTP) involved in the mRNA catalytic process [6], respectively. Translational modulation has been studied focusing on adenylate-uridylate-rich elements (ARE) in the $3^{\prime}$ untranslated regions (UTR) of transcripts. Tumor necrosis factor alpha (Tnf- $\alpha$ ), for example, has been recognized as a primary target of translational inhibition induced by DEX [7-10]. miRNAs induce both positive and negative effects on mRNA stability, as well as translation

*Corresponding author: Katsuhiko Suzuki, Faculty of Sport Sciences, Waseda University, Saitama, Japan, Tel: +81-4-2947-6898; Fax: +81-4-2947-6898; E-mail: katsu.suzu@waseda.jp

Received February 04, 2016; Accepted February 18, 2016; Published February 28, 2016

Citation: Sako H, Suzuki K (2016) Genome-wide Analysis of Acute Inflammatory and Anti-Inflammatory Responses in RAW264 Cells Suggests cis-Elements Associated with Translational Regulation. J Data Mining Genomics Proteomics 7: 191. doi:10.4172/2153-0602.1000191

Copyright: ( 2016 Sako H, et al. This is an open-access article distributed under the terms of the Creative Commons Attribution License, which permits unrestricted use, distribution, and reproduction in any medium, provided the original author and source are credited. 
Citation: Sako H, Suzuki K (2016) Genome-wide Analysis of Acute Inflammatory and Anti-Inflammatory Responses in RAW264 Cells Suggests cisElements Associated with Translational Regulation. J Data Mining Genomics Proteomics 7: 191. doi:10.4172/2153-0602.1000191

Page 2 of 9

efficiency. Although miRNAs generally negatively regulate the stability and/or translation of mRNA [6,11], translational upregulation can be induced by miRNA by forming a micro-ribonucleoprotein complex and targeting ARE $[12,13]$. Despite such critical roles of glucocorticoids on post-transcriptional controls, including translational regulation, the global impact of glucocorticoids on translational dynamics has not been investigated.

It is now possible to examine the differential regulation between transcription and translation by using a genome-wide translation analysis by employing ribosome profiling (Ribo-Seq), a recently developed technology [14]. In Ribo-Seq, translating ribosomes can be stalled by the translational inhibitor cycloheximide (CHX) or flash frozen, and ribosome-protected fragments (RPF) can be extracted, followed by processing the RPF for next generation sequencing [1416]. Ribo-Seq together with a genome-wide transcriptional analysis (mRNA-Seq) enables us to investigate the unique translational regulation that is independent of transcriptional control.

Here, we conducted a genome-wide transcriptional and translational analysis in an acute inflammatory model using RAW264 macrophages stimulated by LPS alone or LPS coupled with DEX (LPS + DEX). When the differentially regulated genes between LPS and LPS + DEX were compared, translational control predominated the differences and the changes in transcription were minor. Motif enrichment analysis on the up- or down-regulated genes at translation levels suggested cis-elements were exclusively enriched in the $3^{\prime}$-UTR of the differentially regulated gene clusters, suggesting that the currently identified cis-elements may be a key to understanding the acute translational responses as well as the complex mechanisms of the anti-inflammatory effect of glucocorticoids.

\section{Materials and Methods}

\section{Accession number}

The raw sequenced and processed files from this article have been deposited in the National Center for Biotechnology Information's Gene Expression Omnibus under the accession number GSE60930.

\section{Cell culture}

RAW264 cells (DS Pharma Biomedical, Osaka, Japan) were cultured at $3.0 \times 10^{5}$ cells $/ \mathrm{mL}$ in media (DMEM, $2 \mathrm{mM}$ Glutamine, $10 \%$ FBS, 100 units penicillin, and $100 \mu \mathrm{g}$ streptomycin $/ \mathrm{mL}$ ) for $24 \mathrm{~h}$ before harvest. The confluency of cells was confirmed to never exceed $80-90 \%$. Cells were stimulated by LPS $(100 \mathrm{ng} / \mathrm{mL})$ or LPS $(100 \mathrm{ng} / \mathrm{mL})+$ DEX $(10 \mu \mathrm{M})$ for $30 \mathrm{~min}$. Before harvesting, CHX (final concentration: 100 $\mu \mathrm{g} / \mathrm{mL}$ ) was added into all the conditions (basal, LPS, and LPS + DEX). Two independent biological replicates for both Ribo-Seq and mRNASeq were prepared.

\section{Sequence library preparation and sequencing}

Sequence libraries for Ribo-Seq and mRNA-Seq were prepared as previously described $[17,18]$ with some modifications to the original protocol $[14,16]$, followed by Ion Total RNA-Seq Kit v2 (Ion Torrent, Life Technologies, Carlsbad, CA) according to the manufacturer's protocols, except that reverse-transcribed cDNA was gel-purified to remove excess primer dimers. The gel region (around $63 \mathrm{nt}$ ) was excised and recovered. Sequencing was conducted by the Ion PGM Template OT2 $200 \mathrm{Kit}$, Ion PGM sequencer, Ion PGM Sequencing 200 Kit v2, and Ion 318 Chip Kit v2, according to the manufacturer's instructions (Ion Torrent).
The alignment for RAW264 sequenced data was conducted by the Ion Torrent server and the reads shorter than $24 \mathrm{nt}$ were trimmed off. For alignment, reference Ensembl genes were retrieved from GRCm38 using the Biomart MartView (http://www.biomart.org/ biomart/martview) [19]. Sequenced reads were aligned to coding DNA sequences (CDS) for quantification and to CDS with sequences $25 \mathrm{nt}$ upstream of the AUG translational start site for metagene analysis.

\section{Sequenced read analysis}

The following analyses were conducted by $\mathrm{R}$ and in-house $\mathrm{R}$ scripts using expressed genes ( $>15$ Reads per Million $[R P M], n=9,620$ ) if not otherwise specified. To validate Ribo-Seq and mRNA-Seq, Pearson correlations were calculated for $\log _{2}$ RPM of each pair of biological replicates. Metagene analysis was carried out with the ribosome counts at a relative distance from the AUG start codon, a nucleotide site of each gene, in which the $5^{\prime}$ end of the RPF was used for alignment. Ribosome counts were normalized by dividing raw counts by the mean counts of the $1-\mathrm{kb}$ region from the start codon.

Differentially regulated genes were detected using edgeR with Trimmed Mean of M (TMM) adjustment [20]. The selected genes (FDR $<0.05)$ in each condition were used to create a heatmap, where $\log _{2}$-transformed RPM ratios were hierarchically clustered by Ward's method in each regulatory cluster. Gene ontology (GO) enrichment analysis was also conducted by DAVID [21,22].

To characterize the stimulated gene responses to LPS or LPS + DEX, feedback genes were retrieved from a previous study [23] and inflammatory response genes were the genes belonging to the GO term "inflammatory response" in Table 1. Translational efficiency was calculated by the division of RPF RPM values by those of mRNA and then box-plotted. The Kolmogorov-Smirnov (KS) test was used to compare statistical differences between translation efficiencies, i.e., inflammatory response genes in basal vs. all genes in basal, inflammatory response genes in LPS vs. all genes in LPS, inflammatory response genes in LPS + DEX vs. all genes in LPS + DEX, feedback genes in basal vs. all genes in basal, feedback genes in LPS vs. all genes in LPS, and feedback genes in LPS + DEX vs. all genes in LPS + DEX.

TOP-like motif genes include the known TOP-motif genes and TOP-like genes defined in a previous study [24]. The translational efficiency ratio was calculated by dividing a translational efficiency in LPS or LPS + DEX conditions by the efficiency in the basal condition.

The putative regulatory cis-elements for the differentially regulated gene group between LPS and LPS + DEX $($ FDR $<0.01)$ were predicted by Multiple Em for Motif Elicitation [25] with a minimum and maximum motif width of 6 and $8 \mathrm{nt}$, respectively. An E-value was used to evaluate the significance of the predicted cis-elements. The two-sided Fisher's exact test was used to confirm the significance of occurrence for the cis-elements in each differentially regulated gene group (FDR $<0.01)$ compared with all the genes analyzed. To avoid the bias from splicing variances, a transcript with the longest $3^{\prime}$-UTR was selected as the representative of the gene throughout the prediction and evaluation processes of cis-elements. Mature miRNA sequences were retrieved from miRBase [26] and screened for the seed sequence (position 2 to 8 from $5^{\prime}$ end of miRNA) complementary to the putative cis-elements.

\section{Results}

We first evaluated the reproducibility and validity of mRNA-Sec and Ribo-Seq in the basal, LPS, and LPS + DEX conditions (Figure 1). Both sequencings showed strong Pearson correlations between 
Citation: Sako H, Suzuki K (2016) Genome-wide Analysis of Acute Inflammatory and Anti-Inflammatory Responses in RAW264 Cells Suggests cisElements Associated with Translational Regulation. J Data Mining Genomics Proteomics 7: 191. doi:10.4172/2153-0602.1000191

Page 3 of 9

individual biological replicates, ranging from $R^{2}=0.93$ to 0.99 (Figure 1A). As previous Ribo-Seq studies reported [14,16], strong peaks with a $12 \mathrm{nt}$ offset were observed at the AUG start codon throughout all the conditions (Figure 1B). Importantly, a clear triplet periodicity was also detected in Ribo-Seq (Figure 1B), an indication of the successful capturing of the dynamics of translating ribosomes with high reproducibility.

To explore the acute effect of either LPS or the combination of LPS and DEX on RAW264 cells, differentially regulated genes were identified using edgeR. We then selected differentially regulated genes (FDR < 0.05) either in transcription or translation levels in LPS or LPS + DEX (Supplementary Table 1-6). Among the genes analyzed ( $\mathrm{n}=9,621)$, the numbers of differentially regulated genes at the transcriptional level were 163 (LPS-induced downregulation), 213 (LPS + DEX-induced downregulation), 35 (LPS -induced upregulation), and 96 (LPS + DEX-induced upregulation). The numbers of translationally regulated genes were 184 (LPS-induced downregulation), 176 (LPS + DEX-induced downregulation), 271 (LPS-induced upregulation), and 157 (LPS + DEX-induced upregulation). To examine the differential regulation between transcription and translation, log-transformed expression ratios (relative to the basal conditions) of the selected genes were clustered and compared at transcription (mRNA) and translation (RPF) levels (Figure 2). In the upregulated mRNA gene group, RPF similarly increased in most of the genes under LPS and LPS + DEX stimulation. This was consistent with the enriched functional similarities in the GO analysis (Tables 1 and 2). GO-terms associated with inflammation were seen in both mRNA Up and RPF Up groups in both LPS and LPS + DEX conditions. However, distinctions were identified in the upregulated RPF genes, exemplified by Fus, mt-Nd5, and mt-Col (Figure 3, Supplementary Figure 1A and 1B), in which RPF increased without enhancing the corresponding transcript levels. Genes related to ribosomes were highly enriched both in LPS and LPS + DEX (RPF up) conditions, which was not seen in mRNA levels (Tables 1 and 2). The translation-dependent upregulation also exhibited differences between LPS and LPS + DEX, represented by the upregulated genes involved in oxidative phosphorylation, cell projection, and translation initiation factor activity in LPS or ATPase activity in LPS + DEX (Tables 1 and 2). In downregulated genes, histone core genes were enriched in both LPS- and LPS + DEX-induced differential expression in RPF, but not in mRNA levels; this was due to the absence of polyA tails in these transcripts, and the fact that we purified only polyA mRNA for mRNA-Seq.

A recent study reports that acute LPS-induced inflammation can lead to the derepression and translational upregulation of the feedback inhibitors of inflammation [23]. We examined the translational dynamics of these feedback genes, as well as inflammatory response genes categorized into a GO term, "inflammatory response", in Table 1 (Supplementary Figures 2A and 2B). The biplots exhibited similar upregulation in the two group genes at both mRNA and RPF levels, suggesting a transcript-driven regulation. In both gene groups, translation efficiency showed slightly declined efficiencies under LPS or LPS + DEX stimulation rather than enhanced efficiency (Supplementary Figure 3). Although the reduced efficiency is inconsistent with the previous study, this nominal decrease can be explained by the rapidly increased mRNA levels (see Discussion).

Previous studies reported that DEX inhibits the translation of inflammatory genes, such as Tnf- $\alpha$ [7-10]. When mRNA and RPF levels were separately observed (Figure 4 ), we found that $T n f-\alpha, C x c l 2$, and Ccl4 exhibited translational downregulation, even though these mRNA expressions were promoted or not changed in LPS + DEX compared with LPS alone. This suggests a robust and acute translational regulation within 30 min of DEX stimulation coupled with LPS.
To capture the effect of LPS + DEX on the global translational landscape, we investigated the differential regulation between LPS and LPS + DEX stimulations (Figure 5). More genes were significantly upor downregulated at the translation level rather than at the transcript level. Although transcriptional regulation showed no downregulation and only 7 upregulated genes, including Dusp 1, 407 genes were significantly decreased and 291 genes were significantly increased at the translation level under the LPS + DEX stimulation rather than LPS alone (Figure 5A). When the regulation of transcription and translation was compared, some genes showed similar expression patterns; however, most of the genes exhibited distinct regulatory features, where genes were translationally up- or downregulated without changing the corresponding mRNA levels (Figure 5B). To further reveal the characteristics of the genes that were exclusively translationally regulated under the LPS + DEX stimulation or with greater extent in LPS + DEX than in LPS alone, GO enrichments were examined (Table 3). Translationally upregulated genes were predominantly enriched in RNA splicing, the ribosome, the cytoskeleton, ATP binding, and the mRNA catabolic process. Translationally downregulated genes were found in oxidative phosphorylation, the histone core, protein localization, the ribosome, translational initiation factor, and the immune response with lesser extent.

DEX has been recognized as a strong translational suppressor by modulating the mTOR signaling cascade. The prevention of mTOR activity attenuates cap-dependent translational initiation and TOPmotif genes, represented by a T/C rich sequence in their 5 '-UTR, are highly prone to mTOR suppression [24]. Therefore, TOP-motif and TOP-like genes can be a reasonable indicator to estimate the inhibitory effect of DEX on mTOR-dependent translation. We analyzed the translational efficiency ratios of TOP-like genes in LPS and LPS + DEX conditions (Figure 6). Although the translation efficiency ratio was higher in TOP-like genes than that in other genes, there was no difference in the efficiencies of TOP-like genes between LPS and LPS + DEX. This suggests a nominal effect of DEX on mTOR-dependent translation within 30 min of LPS + DEX treatment.

To reveal the potential elements responsible for LPS + DEXinduced translational regulation, sequence motifs were screened, focusing on the $3^{\prime}$-UTR of the differentially regulated genes (Figure 7). There were 3 sequence motifs identified, all of which presented significant enrichment scores (Figure 7A). One of these, CCAGCCTG, was enriched in the $3^{\prime}$-UTR of the downregulated gene clusters. The other two motifs, a poly-A stretch and CCCCAGCC, were enriched in the upregulated genes. Because we aimed to identify sequence motifs that were responsible for LPS + DEX-induced translational regulation, and were not involved in LPS-stimulation or transcriptional regulation, the three identified motifs were tested for their enrichment significance in each gene cluster, including translational up- or downregulation under LPS + DEX vs. LPS and LPS vs. Basal conditions, and transcriptional up- or down-regulation under LPS vs. Basal and LPS + DEX vs. Basal conditions (Figure 7B, Supplementary Table 7). To this end, we conducted two-sided Fisher's exact tests after counting the number of exact matches of the sequence motifs in each gene cluster's 3'-UTR sequences. As expected, each of the motifs exhibited significant enrichment only in the original gene cluster from which they were identified (Figure 7B, Supplementary Table 7), supporting that the CCAGCCTG and AAAAAAA/CCCCAGCC motifs might exert exclusive influence on LPS + DEX-induced translational downand upregulation, respectively. 
A

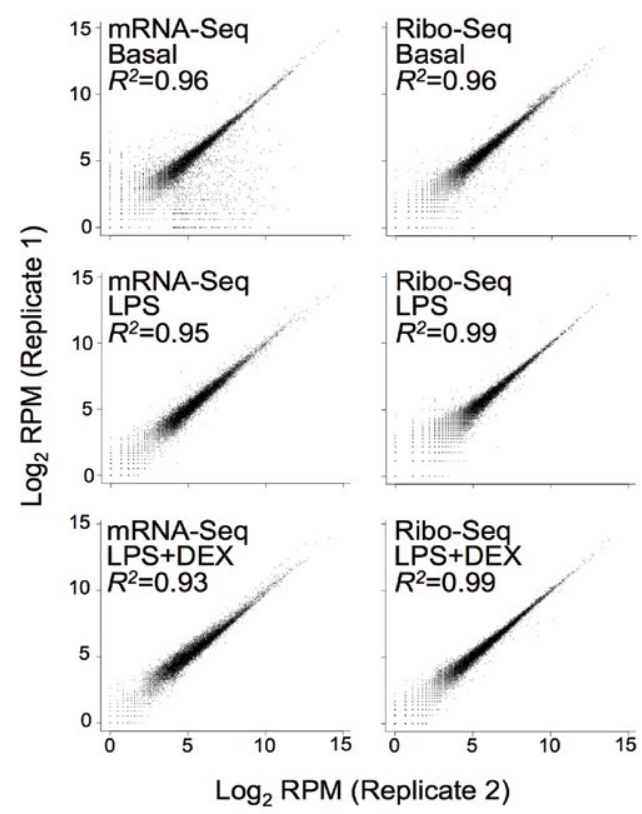

B

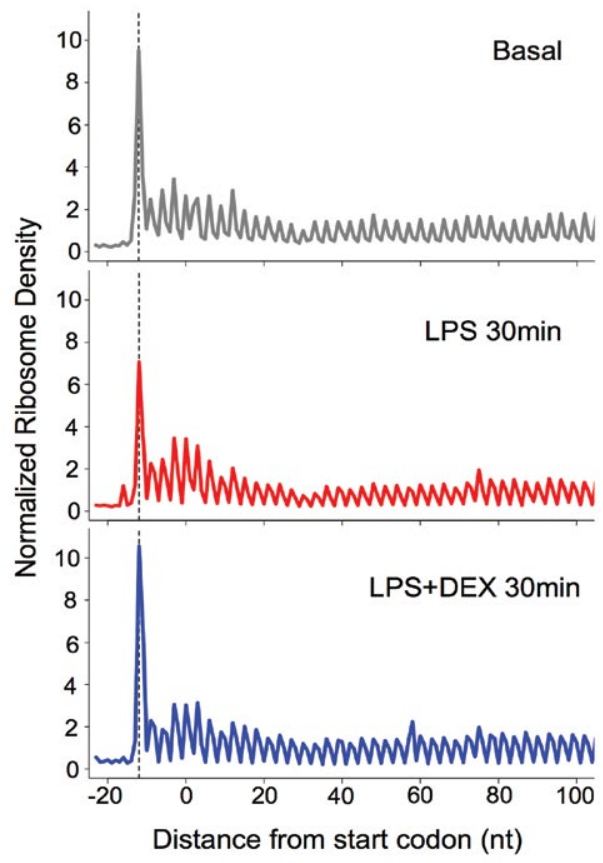

(A) Correlations of $\log _{2}$ expression levels (RPM) in two individual biological replicates of RAW264 for each condition are shown. (B) Metagene analyses near the start codon of analyzed genes are shown. $5^{\prime}$ ends of sequenced reads were aligned and read counts were divided by the mean read counts of the first 1 -kb coding region in each condition. RPM: Reads Per Million.

Figure 1: Both mRNA-Seq and Ribo-Seq showed strong reproducibility and validity.

A

Expression Ratio (LPS vs. Basal)

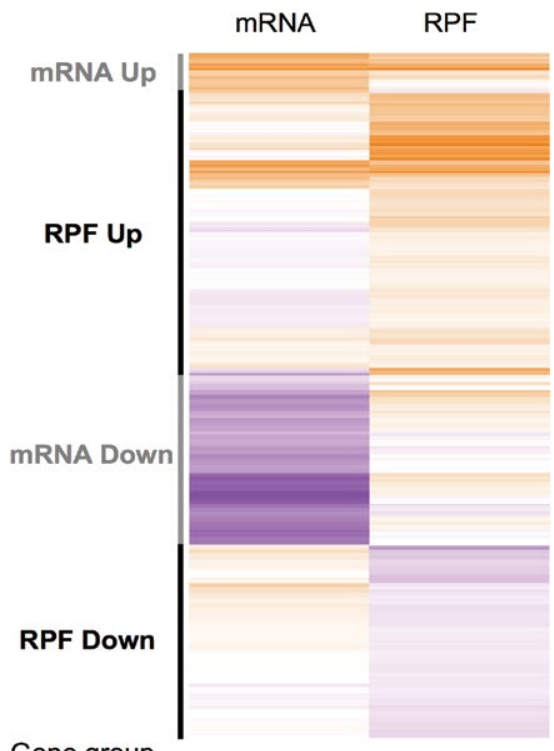

Gene group
B

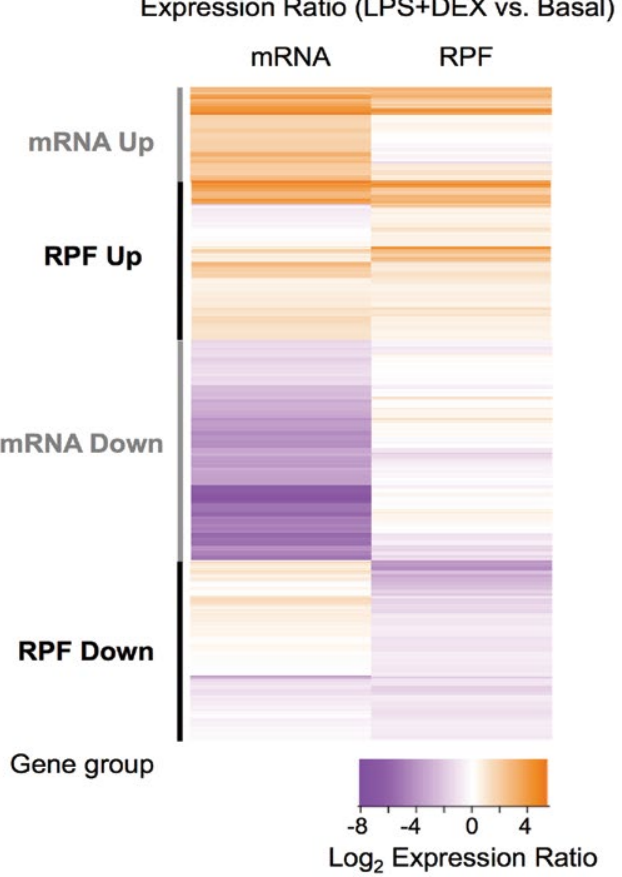

(A) In LPS- and (B) LPS + DEX-stimulated conditions, mRNA and RPF expression patterns are shown by clustering the genes of up- or down-regulated (FDR $<0.05$ ) transcripts (mRNA Up or Down) and translations (RPF Up or Down).

Figure 2: Different gene clusters exhibited distinct expression patters between mRNA and RPF profiles. 
Citation: Sako H, Suzuki K (2016) Genome-wide Analysis of Acute Inflammatory and Anti-Inflammatory Responses in RAW264 Cells Suggests cisElements Associated with Translational Regulation. J Data Mining Genomics Proteomics 7: 191. doi:10.4172/2153-0602.1000191

Fus
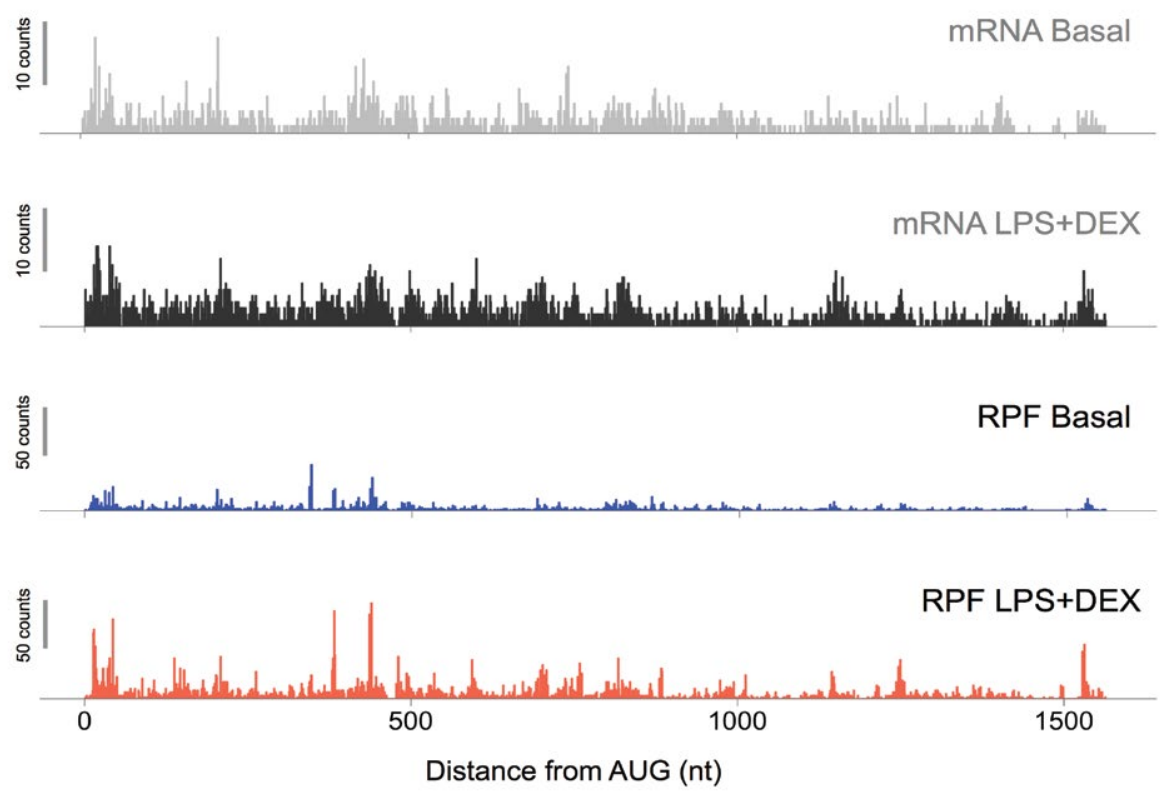

LPS + DEX-induced translational up regulation in Fus. Each expression values were normalized by its total sequenced read counts.

Figure 3: Representative genes regulated in translation levels.

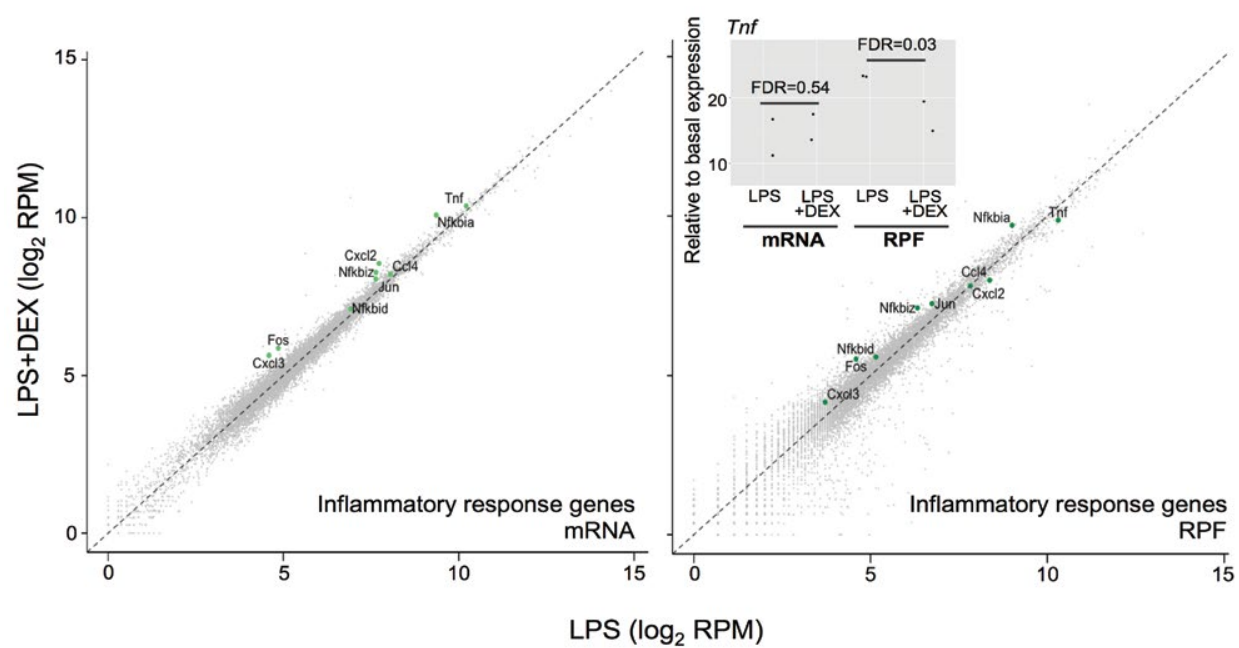

Transcriptional (mRNA) and translational (RPF) responses under LPS and LPS + DEX conditions were compared focusing on the inflammatory response genes. Tnf expression changes at mRNA and RPF levels were further focused by a dot plot. Each black dot represents a biological replicate in mRNA-Seq or Ribo-Seq. FDR: False Discover Rate derived from edgeR outcome.

Figure 4: DEX lowered translation of Tnf and Cc/4 independent to mRNA compared with LPS alone.

Because the $3^{\prime}$-UTR has been known as the target of miRNAtriggered translational suppression, as well as mRNA degradation or even translational upregulation [12,13], we screened miRNA that could target the identified sequence motifs. To this end, miRNA seed sequences ( 2 to $8 \mathrm{nt}$ from the $5^{\prime}$ end of miRNA) were the focus. Four miRNA species were detected, each of which exhibited a perfect complementarity to one of the motifs from their 2 nd seed position to either the 7 th or 8 th position (Figure $7 \mathrm{C}$ ), implying a putative mechanism of LPS + DEX-induced translational regulation.

\section{Discussion}

In the current study, we conducted a genome-wide translational and transcriptional analysis using an acute inflammatory model of RAW264 cells under the stimulation of LPS or LPS coupled with DEX. Overall, the translational dynamics were more striking than those of transcription when LPS and LPS + DEX were compared. The majority of differential regulation was derived from the translation levels. LPS enhanced the translation of the genes associated with oxidative phosphorylation and ribosomes in an mRNA-independent 
Citation: Sako H, Suzuki K (2016) Genome-wide Analysis of Acute Inflammatory and Anti-Inflammatory Responses in RAW264 Cells Suggests cisElements Associated with Translational Regulation. J Data Mining Genomics Proteomics 7: 191. doi:10.4172/2153-0602.1000191

A

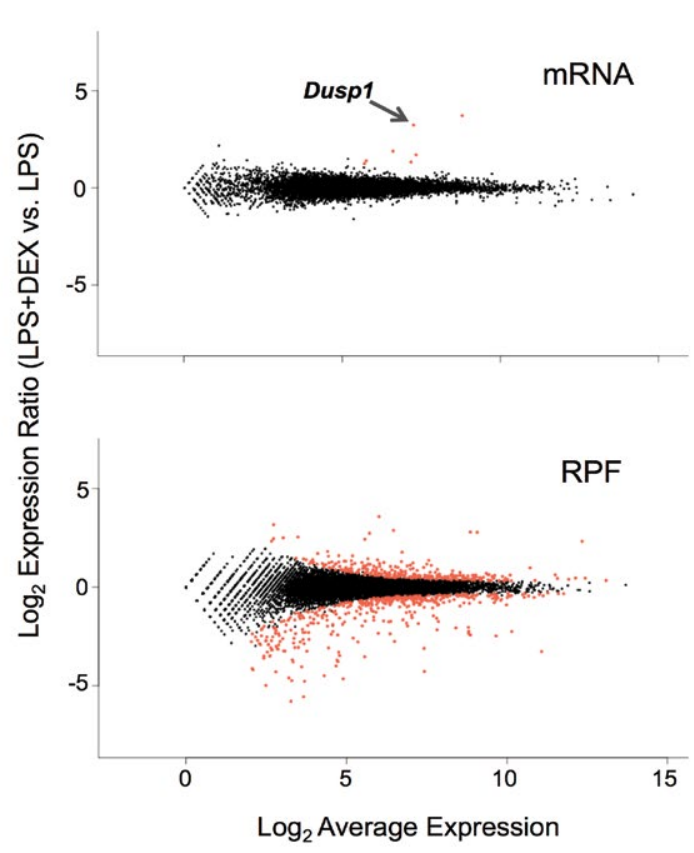

B

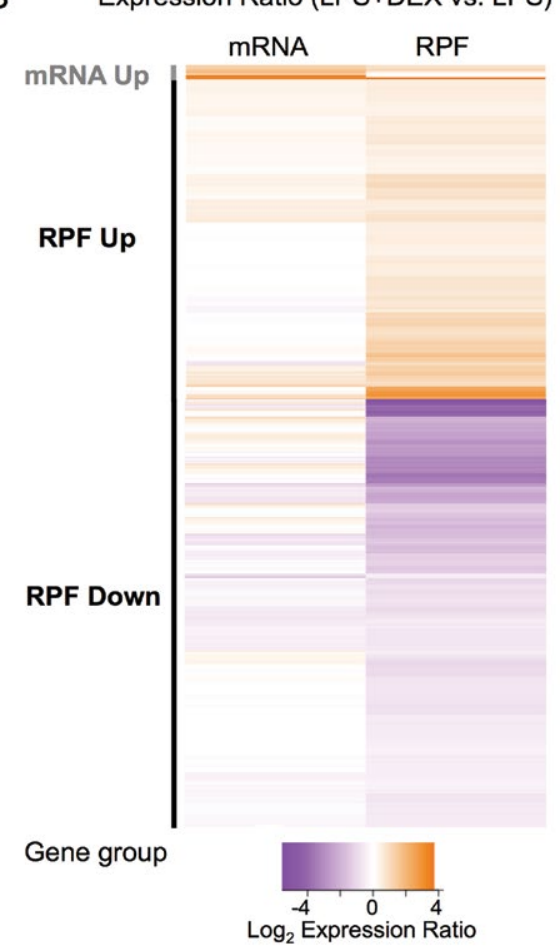

(A) M-A plots for mRNA and RNA expression changes are shown. Differentially regulated genes (FDR < 0.05) are colored in red. A well-known gene, Dusp1, responding to DEX stimuli is indicated in mRNA. (B) Expression patterns in mRNA or RPF are clustered focusing on differentially regulated genes either in transcript (mRNA Up) or translation (RPF Up or Down) by comparing LPS and LPS + DEX (i.e., upregulation refers increased expression in LPS + DEX compared to LPS).

Figure 5: Differences between LPS- and LPS + DEX-stimulation were predominated by translation changes.

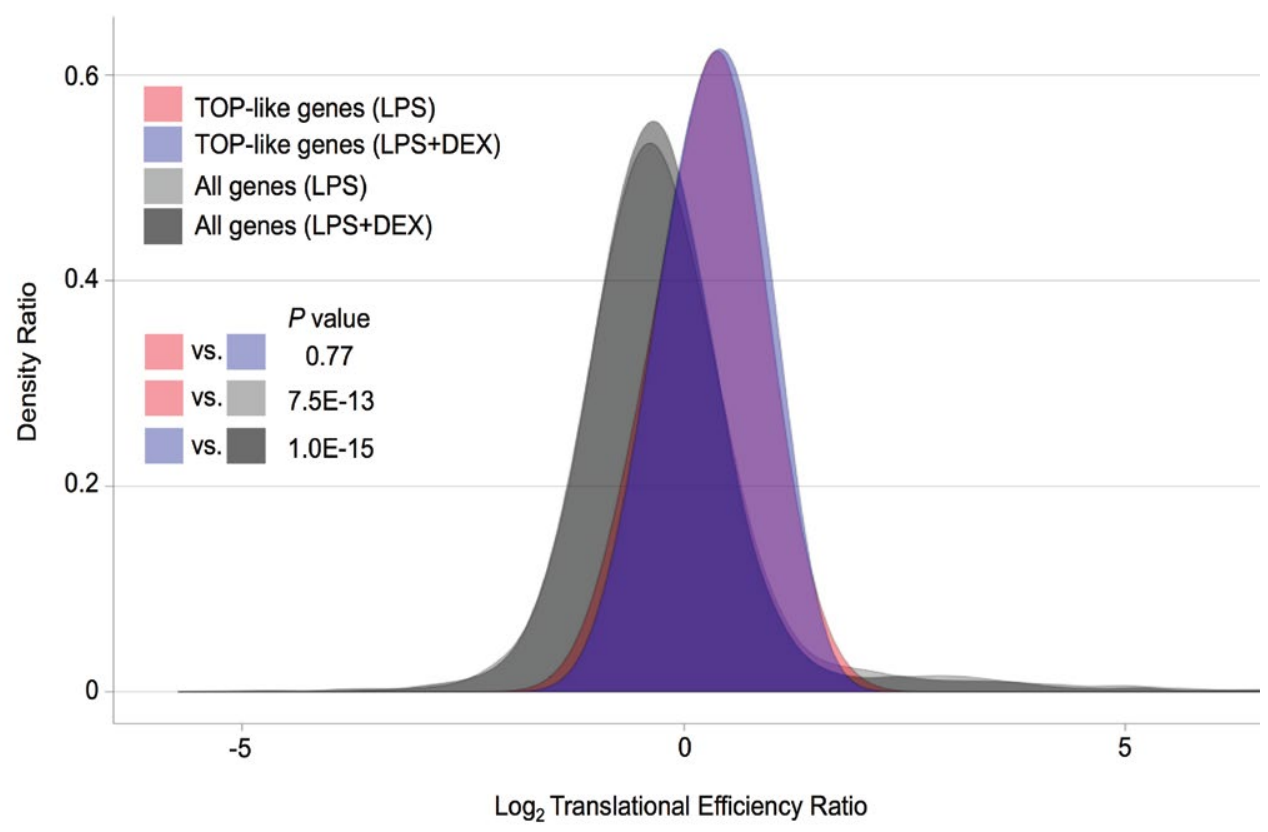

The regulation in translational efficiency in TOP-like genes in LPS and LPS + DEX-stimulated conditions were shown. Statistics was KS-test.

Figure 6: Translation efficiency ratios of TOP-like genes were not altered between LPS and LPS + DEX. 
Citation: Sako H, Suzuki K (2016) Genome-wide Analysis of Acute Inflammatory and Anti-Inflammatory Responses in RAW264 Cells Suggests cisElements Associated with Translational Regulation. J Data Mining Genomics Proteomics 7: 191. doi:10.4172/2153-0602.1000191

A

$\begin{array}{lcl}\text { Gene group } & \text { Sequence } \\ \begin{array}{c}\text { Down regulated } \\ \text { (LSP+DEX vs. LPS) }\end{array} & 3 \text { 'UTR } \\ \begin{array}{c}\text { Up regulated } \\ \text { (LSP+DEX vs. LPS) }\end{array} & 3 \text { 3'UTR } \\ \begin{array}{c}\text { Up regulated } \\ \text { (LSP+DEX vs. LPS) }\end{array} & 3 \text { 3'UTR }\end{array}$

C

\begin{tabular}{|c|c|c|}
\hline $\begin{array}{l}\text { Identified Motif Sequence } \\
\text { miRNA Seed Sequence } \\
\text { miR-7656-3p }\end{array}$ & 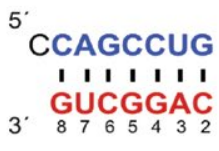 & $\begin{array}{l}3 \\
5\end{array}$ \\
\hline $\begin{array}{l}\text { Identified Motif Sequence } \\
\text { miRNA Seed Sequence }\end{array}$ & $\begin{array}{l}5^{\circ} \text { AAAAAAA } \\
\text { I I } 1 \text { I I I I } \\
\text { CUUUUUUU }\end{array}$ & 3 \\
\hline miR-6951-3p, miR-7116-3p & 3. $\quad 7655432$ & 5 \\
\hline
\end{tabular}

Identified Motif Sequence

miRNA Seed Sequence miR-3083-5p

B

\begin{tabular}{cccccc} 
Motif & Sequence & Regulation & Condition & Gene group & $P$ value \\
\hline CCAGCCTG & $3^{\prime}$ 'UTR & RPF & LPS+DEX vs. LPS & Down regulated & 0.0074 \\
CCAGCCTG & $3^{\prime}$ 'UTR & RPF & LPS+DEX vs. LPS & Up regulated & 1 \\
\hline AAAAAAA & $3^{\prime}$ 'UTR & RPF & LPS+DEX vs. LPS & Down regulated & 0.25 \\
AAAAAAA & $3^{\prime}$ 'UTR & RPF & LPS+DEX vs. LPS & Up regulated & 0.006 \\
\hline CCCCAGCC & $3^{\prime}$ 'UTR & RPF & LPS+DEX vs. LPS & Down regulated & 0.78 \\
CCCCAGCC & $3^{\prime}$ 'UTR & RPF & LPS+DEX vs. LPS & Up regulated & 0.0004 \\
\hline
\end{tabular}

Putative cis-elements and associated miRNAs that may be responsible for the differential translation between LPS and LPS + DEX were predicted. (A) The 3'UTR sequences of differentially translated genes (LPS + DEX vs. LPS) were used to identify enriched motifs specific either down- or up-regulated clusters. MEME targeting 6 to $8 \mathrm{nt}$ motif length was used. (B) Two-sided Fisher's exact test was used to confirm the enrichment of the identified motifs exclusively in either down- or up-regulated gene clusters. (C) miRNAs putatively targeting the identified motifs were screened focusing on miRNA seed sequences ( 2 to 8 nt of 5 ' ends). Each seed sequence position is indicated below the miRNA sequences colored in red.

Figure 7: Putative cis-elements and associated miRNAs were predicted.

\begin{tabular}{|c|c|c|c|}
\hline Regulation & Gene Group & GO term & $\begin{array}{l}\text { Enrichment } \\
\text { Score }\end{array}$ \\
\hline \multirow[t]{9}{*}{ mRNA } & \multirow[t]{5}{*}{ Up (LPS) } & Toll-like receptor signaling pathway & 2.3 \\
\hline & & MAPK aignaling pathway & 2.19 \\
\hline & & $\begin{array}{l}\text { Regulation of transcription factor } \\
\text { activity }\end{array}$ & 2.01 \\
\hline & & Blood vessel development & 1.91 \\
\hline & & Inflammatory response & 1.64 \\
\hline & \multirow[t]{4}{*}{ Down (LPS) } & Cytoskeleton & 2.78 \\
\hline & & $\begin{array}{l}\text { ATPase activity, coupled to } \\
\text { movement of substrates }\end{array}$ & 2.35 \\
\hline & & Cell-cell junction & 1.83 \\
\hline & & Extracellular region & 1.81 \\
\hline \multirow[t]{11}{*}{ RPF } & \multirow[t]{8}{*}{ Up (LPS) } & Ribosome & 5.14 \\
\hline & & Oxidative phosphorylation & 3.73 \\
\hline & & Non-membrane-bound organelle & 2.66 \\
\hline & & Nuclear lumen & 1.82 \\
\hline & & Cell projection & 1.74 \\
\hline & & RNA polymerase & 1.58 \\
\hline & & Inflammatory response & 1.58 \\
\hline & & Translation initiation factor activity & 1.48 \\
\hline & \multirow[t]{3}{*}{ Down (LPS) } & Histone core & 3.21 \\
\hline & & Guanyl nucleotide binding & 2.2 \\
\hline & & Transport & 1.43 \\
\hline
\end{tabular}

Table 1: GO analysis of the LPS-induced differentially regulated genes. manner, which was consistent with a previous study where polysome profiling was applied to LPS-stimulated dendritic cells [27]. This upregulation was attenuated by DEX, suggesting that LPS-induced acute translational upregulation can be blunted, or at least lowered, by the acute translational regulation of DEX as early as within $30 \mathrm{~min}$ of co-stimulation.

It was currently found that DEX-derived translational regulation seems to be independent to mTOR activity. Previous studies have reported that glucocorticoid represses mTOR signaling and the subsequent translational initiation $[10,28,29]$. Given that TOPmotif genes are highly prone to repressed mTOR signaling [24], if a glucocorticoid-induced suppressive effect on mTOR signaling triggered the currently observed DEX-induced translational changes, TOP-motif genes would show a decrease in RPF levels. However, there was no difference between the RPF levels of the TOP-motif genes under LPS or LPS + DEX stimulation. Together with the longer stimulation by DEX in other research $[10,28,29]$, the current result suggests that the $30 \mathrm{~min}$ of DEX stimulation is not sufficient to affect the translation of TOP-motif genes. Although the current observation, DEX-induced translational suppression of the genes involved in translational initiation, agrees with the previous reports $[10,29]$, this regulation appears not to be associated with the attenuation of mTOR signaling. In addition, the effect of glucocorticoids on the mTOR pathway cannot explain the currently observed translational upregulation in LPS + DEX when compared with LPS alone. To suggest an alternative mechanism 
Citation: Sako H, Suzuki K (2016) Genome-wide Analysis of Acute Inflammatory and Anti-Inflammatory Responses in RAW264 Cells Suggests cisElements Associated with Translational Regulation. J Data Mining Genomics Proteomics 7: 191. doi:10.4172/2153-0602.1000191

Page 8 of 9

\begin{tabular}{|c|c|c|c|}
\hline Regulation & Gene Group & GO Term & $\begin{array}{l}\text { Enrichment } \\
\text { Score }\end{array}$ \\
\hline \multirow[t]{10}{*}{ mRNA } & \multirow{7}{*}{$\begin{array}{l}\text { Up (LPS + } \\
\text { DEX) }\end{array}$} & Inflammatory response & 2.4 \\
\hline & & Negative regulation of NF-kappaB & 2.19 \\
\hline & & Blood vessel development & 2.13 \\
\hline & & MAPK aignaling pathway & 1.99 \\
\hline & & mRNA catabolic process & 1.7 \\
\hline & & Regulation of Translation & 1.49 \\
\hline & & Toll-like receptor signaling pathway & 1.38 \\
\hline & \multirow{3}{*}{$\begin{array}{c}\text { Down (LPS + } \\
\text { DEX) }\end{array}$} & Oxidatice phosphorylation & 2.11 \\
\hline & & Cytoskeleton & 2.1 \\
\hline & & $\begin{array}{l}\text { ATPase activity, coupled to } \\
\text { movement of substrates }\end{array}$ & 1.53 \\
\hline \multirow[t]{10}{*}{ RPF } & \multirow{7}{*}{$\begin{array}{l}\text { Up (LPS + } \\
\text { DEX) }\end{array}$} & Non-membrane-bound organelle & 6.15 \\
\hline & & Ribosome & 5.93 \\
\hline & & Blood vessel development & 2.55 \\
\hline & & mRNA catabolic process & 2.15 \\
\hline & & ATPase activity & 1.96 \\
\hline & & Inflammatory response & 1.91 \\
\hline & & $\begin{array}{l}\text { Regulation of transcription factor } \\
\text { activity }\end{array}$ & 1.58 \\
\hline & \multirow{3}{*}{$\begin{array}{c}\text { Down (LPS + } \\
\text { DEX) }\end{array}$} & Histone core & 5.44 \\
\hline & & Oxidative phosphorylation & 2.32 \\
\hline & & Immune response & 1.2 \\
\hline
\end{tabular}

Differentially regulated genes were selected from edgeR $(F D R<0.05)$.

Table 2: GO analysis of the LPS + DEX-induced differentially regulated genes.

\begin{tabular}{|l|l|l|}
\hline Gene Group & GO Term & Enrichment Score \\
\hline \multirow{4}{*}{ RPF Up } & RNA splicing & 4.84 \\
\cline { 2 - 3 } & Ribosome & 3.73 \\
\hline & Cytoskeleton & 3.22 \\
\hline & ATP binding & 2.22 \\
\cline { 2 - 3 } & mRNA catabolic process & 2 \\
\hline & Oxidative phosphorylation & 4.44 \\
\cline { 2 - 3 } & Histone core & 2.56 \\
\cline { 2 - 3 } & Protein localization & 1.81 \\
\cline { 2 - 3 } & Ribosome & 1.47 \\
\cline { 2 - 3 } & Translation initiation factor activity & 1.38 \\
\cline { 2 - 3 } & Immune response & 1.17 \\
\hline \multirow{3}{*}{ RPF Down } & & \\
\hline Differentially & regulated genes were selected & from edgeR (FDR < 0.05). \\
Upregulation & refers greater translation in LPS+DEX than in LPS alone. \\
\hline
\end{tabular}

Table 3: GO analysis of the differentially regulated genes between LPS and LPS + DEX.

during glucocorticoid-induced translational regulation, we carried out cis-element screening and discovered that three motifs (CCAGCCTG, AAAAAAA, and CCCCAGCC) were particularly enriched in the $3^{\prime}$-UTR regions in the differentially regulated genes between LPS and LPS + DEX. Given that miRNA can induce rapid translational regulation prior to the decapping or decay of mRNA [11,30], we showed putative miRNAs whose seed sequences are complementary to the cis-elements. Taken together, these cis-elements in the 3 '-UTR and the associated miRNAs may play a role in modulating glucocorticoidinduced rapid translational regulation.

We observed the decline in translation efficiency, which was probably attributable to the delay in ribosomal loading on newly exported mRNA, as previously described $[23,31,32]$. The inflammatory response genes and feedback genes presented seemingly decreased tendency in translation efficiency in LPS and LPS + DEX conditions. However, given that RPF alone showed significant upregulation in both conditions and that others have addressed such an issue, where acute increases in mRNA levels could lead to a nominal decrease in translation efficiency $[23,31,32]$, the currently observed decrease in translation efficiency could be due to the rapidly enhanced mRNA levels that had just been exported from the nucleus and still had not been loaded by ribosomes.

A related limitation of the current study is that it was not possible to differentiate the types of translational upregulation. Specifically, the current approach is not designed to decipher whether overall translation of a gene increases because the number of loaded ribosomes increases (denser polysomes) or it decreases the unloaded "empty" mRNA (more monosome or disome). We failed to detect the previously discovered regulation that LPS could induce the derepression of inflammatory genes (i.e., shift from monosomes or disomes to heavier polysomes) [23]. Despite the drawback, Ribo-Seq is robust against the unwanted bias coming from RNA-binding proteins or ribosomes attached to $5^{\prime}$ UTR, which may not be incorporated in translation but are likely to adversely affect the accuracy of polysome profiling [14,33]. Therefore, we took advantage of Ribo-Seq to more accurately measure the ribosomal dynamics at a sub-codon resolution.

Using the advantages of Ribo-Seq, we analyzed the different translational regulation between LPS + DEX and LPS alone using an acute inflammatory model of RAW264 cells. Our results suggest that alternative regulatory mechanisms, involving cis-elements specifically enriched in up- or downregulated genes as well as the predicted miRNA species, may modulate the acute translational dynamics induced by glucocorticoids when coupled with LPS stimulation.

\section{Acknowledgement}

This study was supported by grants from the Ministry of Education, Culture, Sports Science and Technology of Japan, a Grant-in-Aid for the Global COE Program "Sport Science for the Promotion of Active Life", Challenging Exploratory Research 26560370, Scientific Research (A) $15 \mathrm{H} 0183$ and the Strategic Research Foundation at Private Universities from the Ministry of Education, Culture, Sports, Science and Technology of Japan. The funding sources had no role in study design, data collection and analysis, decision to publish, or preparation of the manuscript.

\section{References}

1. Beato M, Herrlich $P$, Schutz $G$ (1995) Steroid hormone receptors: many actors in search of a plot. Cell 83: 851-857.

2. Nissen RM, Yamamoto KR (2000) The glucocorticoid receptor inhibits NFkappaB by interfering with serine-2 phosphorylation of the RNA polymerase II carboxy-terminal domain. Genes Dev 14: 2314-2329.

3. Adcock IM, Caramori G (2001) Cross-talk between pro-inflammatory transcription factors and glucocorticoids. Immunol Cell Biol 79: 376-384.

4. Shah S, King EM, Chandrasekhar A, Newton R (2014) Roles for the mitogenactivated protein kinase (MAPK) phosphatase, DUSP1, in feedback control of inflammatory gene expression and repression by dexamethasone. J Biol Chem 289: $13667-13679$

5. Kassel O, Sancono A, Kratzschmar J, Kreft B, Stassen M, et al. (2001) Glucocorticoids inhibit MAP kinase via increased expression and decreased degradation of MKP-1. EMBO J 20: 7108-7116.

6. Stellato C (2012) Posttranscriptional gene regulation: novel pathways for glucocorticoids' anti-inflammatory action. Transl Med UniSa 3: 67-73.

7. Han J, Thompson P, Beutler B (1990) Dexamethasone and pentoxifylline inhibit endotoxin-induced cachectin/tumor necrosis factor synthesis at separate points in the signaling pathway. J Exp Med 172: 391-394.

8. Newton R (2000) Molecular mechanisms of glucocorticoid action: what is important? Thorax 55: 603-613.

9. Swantek JL, Cobb MH, Geppert TD (1997) Jun N-terminal kinase/stress activated protein kinase (JNK/SAPK) is required for lipopolysaccharide stimulation of tumor necrosis factor alpha (TNF-alpha) translation: glucocorticoids inhibit TNF-alpha translation by blocking JNK/SAPK. Mol Cell Biol 17: 6274-6282. 
Citation: Sako H, Suzuki K (2016) Genome-wide Analysis of Acute Inflammatory and Anti-Inflammatory Responses in RAW264 Cells Suggests cisElements Associated with Translational Regulation. J Data Mining Genomics Proteomics 7: 191. doi:10.4172/2153-0602.1000191

10. Beck IM, Vanden Berghe W, Vermeulen L, Yamamoto KR, Haegeman G, et al. (2009) Crosstalk in inflammation: the interplay of glucocorticoid receptor-based mechanisms and kinases and phosphatases. Endocr Rev 30: 830-882.

11. Bazzini AA, Lee MT, Giraldez AJ (2012) Ribosome profiling shows that miR430 reduces translation before causing mRNA decay in zebrafish. Science 336: 233-237.

12. Vasudevan S, Tong $Y$, Steitz JA (2007) Switching from repression to activation: microRNAs can up-regulate translation. Science 318: 1931-1934.

13. Vasudevan S, Steitz JA (2007) AU-rich-element-mediated upregulation of translation by FXR1 and Argonaute 2. Cell 128: 1105-1118.

14. Ingolia NT, Ghaemmaghami S, Newman JR, Weissman JS (2009) Genomewide analysis in vivo of translation with nucleotide resolution using ribosome profiling. Science 324: 218-223.

15. Ingolia NT, Brar GA, Rouskin S, McGeachy AM, Weissman JS (2012) The ribosome profiling strategy for monitoring translation in vivo by deep sequencing of ribosome-protected mRNA fragments. Nat Protoc 7: 1534-1550.

16. Ingolia NT, Lareau LF, Weissman JS (2011) Ribosome profiling of mouse embryonic stem cells reveals the complexity and dynamics of mammalian proteomes. Cell 147: 789-802.

17. Sako H, Suzuki K (2014) Exploring the importance of translational regulation in the inflammatory responses by a genome-wide approach. Exerc Immuno Rev 20: 55-67.

18. Sako H, Yada K, Suzuki K (2016) Genome-wide analysis of acute endurance exercise-induced translational regulation in mouse skeletal muscle. PLOS ONE 11: e0148311.

19. Kasprzyk A (2011) BioMart: driving a paradigm change in biological data management. Database (Oxford) 2011: bar049.

20. Robinson MD, McCarthy DJ, Smyth GK (2010) edgeR: a Bioconductor package for differential expression analysis of digital gene expression data. Bioinformatics 26: 139-140.

21. Huang da W, Sherman BT, Lempicki RA (2009) Systematic and integrative analysis of large gene lists using DAVID bioinformatics resources. Nat Protoc 4: 44-57
22. Huang da W, Sherman BT, Lempicki RA (2009) Bioinformatics enrichment tools: paths toward the comprehensive functional analysis of large gene lists. Nucleic Acids Res 37: 1-13.

23. Schott J, Reitter S, Philipp J, Haneke K, Schafer H, et al. (2014) Translational regulation of specific mRNAs controls feedback inhibition and survival during macrophage activation. PLOS Genet 10: e1004368.

24. Thoreen CC, Chantranupong L, Keys HR, Wang T, Gray NS, et al. (2012) A unifying model for mTORC1-mediated regulation of mRNA translation. Nature 485: 109-113.

25. Bailey TL, Elkan C (1994) Fitting a mixture model by expectation maximization to discover motifs in biopolymers. Proc Int Conf Intell Syst Mol Biol 2: 28-36.

26. Griffiths-Jones S (2004) The microRNA registry. Nucleic Acids Res 32: D109-11.

27. Ceppi M, Clavarino G, Gatti E, Schmidt EK, de Gassart A, et al. (2009) Ribosomal protein mRNAs are translationally-regulated during human dendritic cells activation by LPS. Immunome Res 5: 5 .

28. Wang H, Kubica N, Ellisen LW, Jefferson LS, Kimball SR (2006) Dexamethasone represses signaling through the mammalian target of rapamycin in muscle cells by enhancing expression of REDD1. J Biol Chem 281: 39128-39134.

29. Shah OJ, Anthony JC, Kimball SR, Jefferson LS (2000) 4E-BP1 and S6K1 translational integration sites for nutritional and hormonal information in muscle. Am J Physiol Endocrinol Metab 279: E715-E729.

30. Djuranovic S, Nahvi A, Green R (2012) miRNA-mediated gene silencing by translational repression followed by mRNA deadenylation and decay. Science 336: $237-240$.

31. Deneke C, Rudorf S, Valleriani A (2012) Transient phenomena in gene expression after induction of transcription. PLOS ONE 7: e35044.

32. Melamed D, Eliyahu E, Arava Y (2009) Exploring translation regulation by global analysis of ribosomal association. Methods 48: 301-305.

33. Meijer HA, Thomas AA (2002) Control of eukaryotic protein synthesis by upstream open reading frames in the 5 -untranslated region of an mRNA Biochem J 367: 1-11. 